\title{
Inbreeding coefficient and effective size for an X-linked locus in nonrandom mating populations
}

\author{
JINLIANG WANG \\ College of Animal Science, Zhejiang Agricultural University, Hangzhou 310029, China
}

\begin{abstract}
Formulae are given for equilibrium inbreeding coefficients for an X-linked locus in infinite populations under partial full-sib, half-sib and parent-offspring matings, respectively. An exact recurrence equation for the inbreeding coefficient for an X-linked locus is derived for a finite population with equal numbers of males and females under partial full-sib mating. Following the approach of variance of change in gene frequency, two general equations for effective size for an X-linked locus are obtained. The equations consider an arbitrary distribution of family size, unequal numbers of males and females and nonrandom mating. For some special cases, the equations reduce to the simple expressions derived by previous authors. Comparisons are made between expressions for effective size for an X-linked locus and those for an autosomal locus. Some interesting conclusions are drawn from the analysis and discussed.
\end{abstract}

Keywords: effective population size, full-sib mating, genetic drift, heterozygosity, inbreeding.

\section{Introduction}

Since the introduction of the concept of effective population size, much work has been undertaken on this parameter which is important in population and quantitative genetics. Most previous work has been concerned with autosomal genes, and equations for effective size of a wide generality with regard to family size variance, nonrandom mating, unequal numbers for separate sexes and selection have been given in recent years (Caballero, 1994). The study of inbreeding and effective size for X-linked genes and genes in haplodiploid species, however, has been a neglected area until relatively recently. With $\mathrm{X}$-linked loci the situation is rather complex compared to autosomal loci. The heterogametic sex has only one copy of the sex-linked gene, thus the inbreeding coefficient for X-linked loci only refers to the homogametic sex.

For random mating and Poisson distribution of family size, Wright (1933) and Malécot (1951) used path coefficients and identity by descent, respectively, to derive a recurrence equation for the inbreeding coefficient for X-linked loci, and from the equation they deduced the formula for effective size. More general equations for effective size have been obtained which consider an arbitrary family size distribution (Moran \& Watterson, 1959; Ethier \& Nagylaki, 1980; Nagylaki, 1981, 1995; Caballero, 1995) and overlapping generations (Pollak, 1980, 1990) following different approaches. In this paper, I broaden the scope of the previous inquiries by considering nonrandom populations, and not restricting attention to a Poisson distribution of family size. First, I consider the equilibrium inbreeding coefficient for an X-linked locus in infinite populations under different partial inbreeding systems. Secondly, I derive a general recurrence equation for the inbreeding coefficient for an X-linked locus in a finite population with partial full-sib mating. The equation is useful for predicting exact inbreeding coefficients in any generation, especially in early generations when the rate of inbreeding has not reached the asymptotic value. From the recurrence equation I deduce the equation for effective size in partial full-sib mating populations, and then extend it to a more general expression which applies to any nonrandom mating population. Thirdly, following the variance of change in gene frequency approach, I derive two more general equations for effective size accounting for different numbers of males and females, an arbitrary distribution of family size and nonrandom mating. Throughout I assume stable population size, discrete generations, X-linked genes 
or genes in a haplodiploid species which do not affect viability or reproductive ability so that natural selection is not operating to eliminate them.

\section{Theory}

\section{Equilibrium inbreeding coefficient in an infinite population with partial inbreeding}

In deriving recurrence equations for the inbreeding coefficient, it is convenient to use Malécot's 'coefficient de parenté' (Malécot, 1948), which is translated as coefficient of parentage (Kempthorne, 1957) or coancestry (Falconer, 1989). This coefficient may be defined as the probability that two genes at a given locus, one taken at random from each of two randomly selected individuals in the relevant population, are identical by descent. The basic rules for this coefficient have been given by Plum (1954) for an autosomal locus. For an X-linked locus, however, these rules should be adapted because males and females should be distinguished in the pedigree. For the sake of brevity, the heterogametic sex will be referred to as male in this and the next sections.

Consider the general pedigree in Fig. 1. A and C are male parents and $\mathrm{B}$ and $\mathrm{D}$ are female parents. If $\mathrm{P}$ and $\mathrm{Q}$ are both female offspring, then the coancestry of $\mathrm{P}$ with $\mathrm{Q}, G_{\mathrm{PQ}}$, is

$G_{\mathrm{PQ}}=\frac{1}{4}\left(G_{\mathrm{AC}}+G_{\mathrm{AD}}+G_{\mathrm{BC}}+G_{\mathrm{BD}}\right)$,

as is the relation for an autosomal locus. Noting that a gene in a male descends necessarily from a female, whereas one in a female is equally likely to have come from a male or a female, we obtain

$G_{\mathrm{PQ}}=\frac{1}{2}\left(G_{\mathrm{BC}}+G_{\mathrm{BD}}\right)$

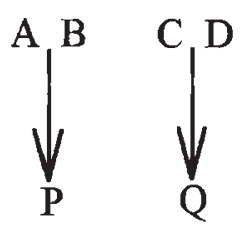

Fig. 1 A general pedigree. if $\mathrm{P}$ is a male and $\mathrm{Q}$ is a female, and

$G_{\mathrm{PQ}}=G_{\mathrm{BD}}$

if both $\mathrm{P}$ and $\mathrm{Q}$ are males.

Now consider the coancestry of an individual, say $\mathrm{P}$, with itself. Similar reasoning yields

$G_{\mathrm{PP}}=\frac{1}{2}\left(1+f_{\mathrm{P}}\right)$

if $\mathrm{P}$ is a female, where $f_{\mathrm{P}}$ is the inbreeding coefficient of $P$, and

$G_{\mathrm{PP}}=1$

if $P$ is a male.

Using these relations we can derive the inbreeding coefficient at equilibrium in an infinite population under the following partial inbreeding systems.

Parent-offspring There are two configurations for parent-offspring mating: father-daughter and mother-son. The pedigrees are shown in Fig. 2(a,b), where circles and squares represent females and males, respectively, and lines and dotted lines represent X-linked genes that are transmitted or not, respectively. For the father-daughter mating, $f_{\mathrm{Z}}=G_{\mathrm{XY}}=\frac{1}{2}\left(G_{\mathrm{XX}}+G_{\mathrm{XW}}\right)=\frac{1}{2}\left(1+f_{t+1}\right)$. If we assume that the proportion of father-daughter matings is $\beta$, then $(\beta / 2)\left(1+f_{t+1}\right)=f_{t+2}$. At equilibrium when $f_{t+1}=f_{t+2}=F_{\mathrm{IS}}$, we have

$F_{\mathrm{IS}}=\beta /(2-\beta)$.

Similarly, we can derive eqn 6 for partial motherson mating. Eqn 6 is a well-known result for partial selfing populations (e.g. Wright, 1969).

Full-sibs Following the approach above, given the pedigree shown in Fig. 2(c), we have $f_{\mathrm{Z}}=G_{\mathrm{XY}}=\frac{1}{2}\left(G_{\mathrm{UW}}+G_{\mathrm{WW}}\right), f_{t+2}=(\beta / 2)\left[f_{t+1}+\left(1+f_{t}\right) / 2\right]$ where $\beta$ is the proportion of full-sib matings, and

$F_{\text {IS }}=\beta /(4-3 \beta)$.

Eqn 7 was also obtained for autosomal genes by many authors (e.g. Ghai, 1969; Li, 1976; Hedrick \& Cockerham, 1986).
Generation

(a)

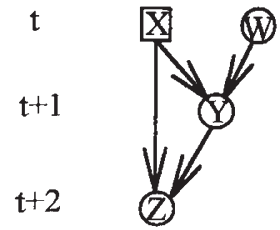

(b)

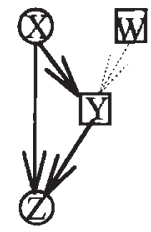

(c)

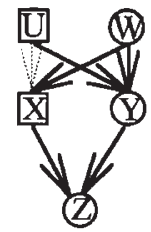

(d)

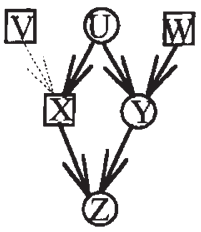

(e)

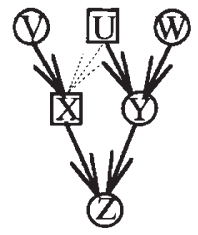

Fig. 2 Pedigrees for matings between different relatives, with circles and squares representing females and males: (a) father-daughter; (b) mother-son; (c) full-sibs; (d) maternal half-sibs; (e) paternal half-sibs. 
Half-sibs Maternal and paternal half-sibs should be distinguished for partial half-sib mating (pedigrees in Fig. 2d,e). For the first case, with the proportion of half-sib matings being $\beta$, we have $f_{\mathrm{Z}}=G_{\mathrm{XY}}=\frac{1}{2}\left(G_{\mathrm{UU}}+G_{\mathrm{UW}}\right), f_{t+2}=(\beta / 2)\left[f_{t+1}+\left(1+f_{t}\right) / 2\right]$ and $F_{\mathrm{IS}}=\beta /(4-3 \beta)$, the same as for partial full-sib mating.

The latter case is a little complicated because the coancestry between $\mathrm{V}$ and $\mathrm{W}$ is difficult to define. If we assume that $\beta$ is small so that $G_{\mathrm{Vw}} \approx 0$, then we get $F_{\text {IS }}=0$.

\section{Equations for the inbreeding coefficient in} populations with equal numbers of males and females

In this section, I consider recurrence equations for the inbreeding coefficient and equations for effective size in finite populations of equal numbers of males and females under partial full-sib mating.

Inbreeding coefficient If we assume that, of the $N / 2$ mating pairs formed from the $N$ individuals (half of each sex) in each generation, $\beta$ is the proportion of full-sib matings, then the proportion of nonsib matings is $1-\beta$. As has been shown previously for the autosomal case (Wang, 1995), the probability that a pair of male and female individuals are fullsibs given that they are not mated is $[2(1+\theta-\beta) N-4 \theta] /[N(N-2)]$, where $\theta$ is the covariance between the numbers of male and female offspring per family. The probability that a random pair of male (female) individuals are full-sibs is $2 \sigma_{\mathrm{m}}^{2} / N\left(2 \sigma_{\mathrm{f}}^{2} / N\right)$, where $\sigma_{\mathrm{m}}^{2}\left(\sigma_{\mathrm{f}}^{2}\right)$ is the variance of the number of male (female) progeny per family.

Let $x, y$ and $z$ represent the coancestry between males, females and a male with a female, and $z_{\mathrm{FS}, t-1}$ $\left(z_{\mathrm{NS}, t-1}\right)$ be the coancestry between a random pair of full-sibs (nonsibs) in separate sexes in generation $t-1$. Similar symbolization applies to $x$ and $y$. Then the average inbreeding coefficient in generation $t$ is

$f_{t}=z_{t-1}=\beta z_{\mathrm{FS}, t-1}+(1-\beta) z_{\mathrm{NS}, t-1}$.

The pedigrees for full-sibs and nonsibs of the same sex or both sexes are diagrammed in Fig. $3(\mathrm{a}-\mathrm{c})$. For full-sib mating (Fig. 3a), using eqns 2 and 4, we obtain the coancestry of M1 with F1

$$
\begin{aligned}
z_{\mathrm{FS}, t-1} & =\frac{1}{2}\left(z_{\mathrm{M} 2 \mathrm{~F} 2}+y_{\mathrm{F} 2 \mathrm{~F} 2}\right)=\frac{1}{2}\left[z_{t-2}+\frac{1}{2}\left(1+f_{\mathrm{F} 2}\right)\right] \\
& =\frac{1}{4}\left(1+2 f_{t-1}+f_{t-2}\right) .
\end{aligned}
$$

For nonsibmating (Fig. 3a), we have
Generation

(a) Male with female

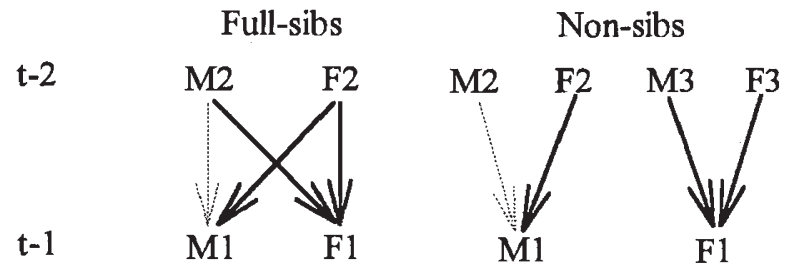

Generation

(b) Two females

$\mathrm{t}-2$

Full-sibs
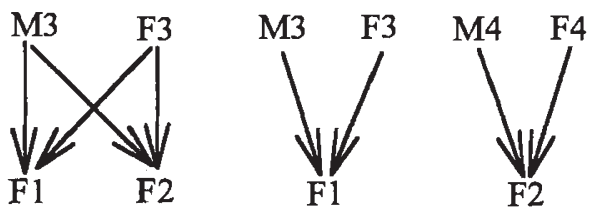

Generation

(c) Two males

Full-sibs

$\mathrm{t}-2$
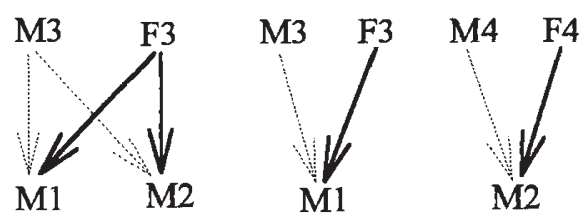

Fig. 3 Pedigrees for full-sibs and nonsibs: (a) male with female; (b) two females; (c) two males.

$$
\begin{aligned}
& z_{\mathrm{NS}, t-1} \\
& =\frac{1}{2}\left(z_{\mathrm{M} 3 \mathrm{~F} 2}+y_{\mathrm{F} 2 \mathrm{~F} 3}\right) \\
& =\frac{1}{2}\left[\frac{2(1+\theta-\beta) N-4 \theta}{N(N-2)} z_{\mathrm{FS}, t-2}\right. \\
& \left.\quad+\frac{N^{2}-2(2+\theta-\beta) N+4 \theta}{N(N-2)} z_{\mathrm{NS}, t-2}\right]+\frac{1}{2} y_{t-2} \cdot(8 \mathrm{c})
\end{aligned}
$$

The coancestry between two females selected at random (Fig. 3b) is

$$
\begin{aligned}
y_{t-1}= & y_{\mathrm{F} 1 \mathrm{~F} 2}=\frac{2 \sigma_{\mathrm{f}}^{2}}{N} y_{\mathrm{FS}, t-1}+\frac{N-2 \sigma_{\mathrm{f}}^{2}}{N} y_{\mathrm{NS}, t-1} \\
= & \frac{2 \sigma_{\mathrm{f}}^{2}}{4 N}\left(2 z_{\mathrm{M} 3 \mathrm{~F} 3}+x_{\mathrm{M} 3 \mathrm{M} 3}+y_{\mathrm{F} 3 \mathrm{~F} 3}\right) \\
& +\frac{N-2 \sigma_{\mathrm{f}}^{2}}{4 N}\left(z_{\mathrm{M} 3 \mathrm{~F} 4}+z_{\mathrm{M} 4 \mathrm{~F} 3}+x_{\mathrm{M} 3 \mathrm{M} 4}+y_{\mathrm{F} 3 \mathrm{~F} 4}\right)
\end{aligned}
$$




$$
\begin{aligned}
= & \frac{2 \sigma_{\mathrm{f}}^{2}}{4 N}\left[2 f_{t-1}+1+\frac{1}{2}\left(1+f_{\mathrm{F} 3}\right)\right]+\frac{N-2 \sigma_{\mathrm{f}}^{2}}{4 N} \\
& \times\left[x_{t-2}+y_{t-2}+\frac{4(1+\theta-\beta) N-8 \theta}{N(N-2)} z_{\mathrm{FS}, t-2}\right. \\
& \left.+\frac{2 N^{2}-4(2+\theta-\beta) N+8 \theta}{N(N-2)} z_{\mathrm{NS}, t-2}\right] \\
& \frac{\sigma_{\mathrm{f}}^{2}}{4 N}\left(3+4 f_{t-1}+f_{t-2}\right)+\frac{N-2 \sigma_{\mathrm{f}}^{2}}{4 N}\left(x_{t-2}+y_{t-2}\right) \\
+ & \frac{N-2 \sigma_{\mathrm{f}}^{2}}{2 N}\left[\frac{2(1+\theta-\beta) N-4 \theta}{N(N-2)} z_{\mathrm{FS}, t-2}\right. \\
& \left.+\frac{N^{2}-2(2+\theta-\beta) N+4 \theta}{N(N-2)} z_{\mathrm{NS}, t-2}\right] .
\end{aligned}
$$

The coancestry between two randomly selected males (Fig. 3c) is

$$
\begin{aligned}
x_{t-1} & =x_{\mathrm{M} 1 \mathrm{M} 2}=\frac{2 \sigma_{\mathrm{m}}^{2}}{N} x_{\mathrm{FS}, t-1}+\frac{N-2 \sigma_{\mathrm{m}}^{2}}{N} x_{\mathrm{NS}, t-1} \\
& =\frac{2 \sigma_{\mathrm{m}}^{2}}{N} y_{\mathrm{F} 3 \mathrm{~F} 3}+\frac{N-2 \sigma_{\mathrm{m}}^{2}}{N} y_{\mathrm{F} 3 \mathrm{~F} 4} \\
& =\frac{\sigma_{\mathrm{m}}^{2}}{N}\left(1+f_{t-2}\right)+\frac{N-2 \sigma_{\mathrm{m}}^{2}}{N} y_{t-2} .
\end{aligned}
$$

When eqns $8 \mathrm{a}-\mathrm{e}$, making proper adaptation to generations, are substituted into each other, we obtain, after some algebra, the general recurrence equation for inbreeding coefficient for an X-linked locus

$$
\begin{aligned}
f_{t}= & \frac{1}{32 N^{3}(N-2)}\left\{2 \left[2(1-\beta)\left(\sigma_{\mathrm{f}}^{2}+2\right)+2 \sigma^{2}+2 \sigma_{\mathrm{f}}^{2}\right.\right. \\
& \left.-\beta \sigma_{\mathrm{m}}^{2}\right] N^{3}-4\left[\sigma_{\mathrm{m}}^{2}+2(3-\beta) \sigma_{\mathrm{f}}^{2}+(2-\beta) \sigma_{\mathrm{m}}^{2} \sigma_{\mathrm{f}}^{2}+4 \theta\right. \\
& \left.-\sigma_{\mathrm{m}}^{2} \theta\right] N^{2}+8 \sigma_{\mathrm{m}}^{2}\left(\sigma_{\mathrm{f}}^{2}-\theta-\theta \sigma_{\mathrm{f}}^{2}\right) N+16 \sigma_{\mathrm{m}}^{2} \sigma_{\mathrm{f}}^{2} \theta \\
& +8 N^{2}\left[(3+2 \beta) N^{2}-2\left(5+\sigma_{\mathrm{f}}^{2}+2 \theta\right) N+4 \sigma_{\mathrm{f}}^{2}\right. \\
& +8 \theta] f_{t-1}+4 N\left[(3-\beta) N^{3}-2\left(2+2 \sigma_{\mathrm{m}}^{2}+\sigma_{\mathrm{f}}^{2}-\theta\right.\right. \\
& \left.+\beta \sigma_{\mathrm{f}}^{2}\right) N^{2}+4\left(2 \sigma^{2}-5 \theta+2 \sigma_{\mathrm{m}}^{2} \sigma_{\mathrm{f}}^{2}+\theta \sigma_{\mathrm{f}}^{2}\right) N
\end{aligned}
$$

$$
\begin{aligned}
& \left.-8 \sigma_{\mathrm{f}}^{2}\left(2 \sigma_{\mathrm{m}}^{2}+\theta\right)\right] f_{t-2}-2\left[(2+6 \beta) N^{4}-2\left(7+2 \sigma^{2}\right.\right. \\
& \left.+\sigma_{\mathrm{f}}^{2}+\theta+\beta+2 \beta \sigma_{\mathrm{m}}^{2}+3 \beta \sigma_{\mathrm{f}}^{2}\right) N^{3}+4\left(4 \sigma^{2}+2 \sigma_{\mathrm{f}}^{2}\right. \\
& \left.-3 \theta+2 \theta \sigma_{\mathrm{m}}^{2}+3 \theta \sigma_{\mathrm{f}}^{2}+2 \sigma_{\mathrm{m}}^{2} \sigma_{\mathrm{f}}^{2}+2 \beta \sigma_{\mathrm{m}}^{2} \sigma_{\mathrm{f}}^{2}\right) N^{2} \\
& \left.-16(2+\theta) \sigma_{\mathrm{m}}^{2} \sigma_{\mathrm{f}}^{2} N-8 \theta\left(2 \sigma_{\mathrm{m}}^{2}+3 \sigma_{\mathrm{f}}^{2}\right) N+32 \sigma_{\mathrm{m}}^{2} \sigma_{\mathrm{f}}^{2} \theta\right] f_{t-3} \\
& -\left[\beta N^{4}-2\left(2 \beta-1+2 \sigma_{\mathrm{m}}^{2}-2 \beta \sigma_{\mathrm{m}}^{2}+\beta \sigma_{\mathrm{f}}^{2}-\theta\right) N^{3}\right. \\
& -4\left(\sigma_{\mathrm{f}}^{2}+\theta+\theta \sigma_{\mathrm{f}}^{2}-2 \beta \sigma_{\mathrm{f}}^{2}-2 \sigma_{\mathrm{m}}^{2} \sigma_{\mathrm{f}}^{2}+2 \beta \sigma_{\mathrm{m}}^{2} \sigma_{\mathrm{f}}^{2}\right) N^{2} \\
& \left.+8\left(2 \sigma_{\mathrm{m}}^{2}+\sigma_{\mathrm{f}}^{2}+2 \sigma_{\mathrm{m}}^{2} \sigma_{\mathrm{f}}^{2}\right) \theta N-32 \sigma_{\mathrm{m}}^{2} \sigma_{\mathrm{f}}^{2} \theta\right] f_{t-4} \\
& \left.+\left(N-2 \sigma_{\mathrm{m}}^{2}\right)\left(N-2 \sigma_{\mathrm{f}}^{2}\right)\left[\beta N^{2}-2(1+\theta) N+4 \theta\right] f_{t-5}\right\},
\end{aligned}
$$

where $\sigma^{2}=\sigma_{\mathrm{m}}^{2}+2 \theta+\sigma_{\mathrm{f}}^{2}$. It is evident that $f_{0}=f_{1}=0$, $f_{2}=\beta / 4$, and $f_{t}(t \geq 3)$ can be predicted using eqn 9 .

For the following two cases, eqn 9 can be reduced considerably.

(1) If the numbers of male and female progeny are independently Poisson distributed, $\sigma_{\mathrm{m}}^{2}=\sigma_{\mathrm{f}}^{2}=1$, $\theta=0, \sigma^{2}=2$, and we get

$$
\begin{aligned}
f_{t}= & \frac{1}{32 N^{2}(N-2)}\left\{2(12-7 \beta) N^{2}-12(3-\beta) N+8\right. \\
& +8 N\left[(3+2 \beta) N^{2}-12 N+4\right] f_{t-1}+4\left[(3-\beta) N^{3}\right. \\
& \left.-2(5+\beta) N^{2}+24 N-16\right] f_{t-2}-4\left[(1+3 \beta) N^{3}\right. \\
& \left.-6(2+\beta) N^{2}+4(6+\beta) N-16\right] f_{t-3}-N\left[\beta N^{2}\right. \\
& \left.-2(1+\beta) N+4] f_{t-4}+(N-2)^{2}(\beta N-2) f_{t-5}\right\} .
\end{aligned}
$$

Eqn 10 again reduces to

$$
\begin{aligned}
f_{t}=\frac{1}{8 N^{2}} & {\left[6 N-4+2 N(3 N-2) f_{t-1}\right.} \\
& \left.\quad+\left(3 N^{2}-6 N+8\right) f_{t-2}-(N-2)^{2} f_{t-3}\right]
\end{aligned}
$$

for random mating $(\beta=2 / N)$, which was obtained by Wright (1933), and

$$
\begin{aligned}
f_{t}= & \frac{1}{16 N^{2}(N-2)}\left[12 N^{2}-18 N+4+4 N\left(3 N^{2}\right.\right. \\
& -12 N+4) f_{t-1}+2\left(3 N^{3}-10 N^{2}+24 N-16\right) f_{t-2} \\
& -2\left(N^{3}-12 N^{2}+24 N-16\right) f_{t-3}+N(N-2) f_{t-4} \\
& \left.-(N-2)^{2} f_{t-5}\right]
\end{aligned}
$$

if full-sib matings are avoided $(\beta=0)$.

(2) If one male and one female progeny are selected at random from each family, $\sigma_{\mathrm{m}}^{2}=\sigma_{\mathrm{f}}^{2}=\theta=\sigma^{2}=0$, and eqn 9 is simplified to

$f_{t}=\frac{1}{32 N(N-2)}\left\{8(1-\beta) N+8 N[(3+2 \beta) N-10] f_{t-1}\right.$

(C) The Genetical Society of Great Britain, Heredity, 76, 569-577. 


$$
\begin{aligned}
& +4 N[(3-\beta) N-4] f_{t-2}-4 N[(1+3 \beta) N-7-\beta] f_{t} \\
& \left.-N[\beta N+2-4 \beta] f_{t-4}+N(\beta N-2) f_{t-5}\right\}
\end{aligned}
$$

For random mating and nonsib mating, eqn 13 reduces to

$f_{t}=\frac{1}{8 N}\left[2+6 N f_{t-1}+3 N f_{t-2}-(N+1) f_{t-3}-f_{t-4}\right]$

and

$$
\begin{aligned}
f_{t}= & \frac{1}{16(N-2)}\left[4+4(3 N-10) f_{t-1}\right. \\
& \left.+2(3 N-4) f_{t-2}-2(N-7) f_{t-3}-f_{t-4}-f_{t-5}\right],
\end{aligned}
$$

respectively.

Effective size Using a procedure shown previously (Wang, 1995), an expression for the effective size, $N_{\mathrm{e}}$, can be obtained from eqn 9 . Ignoring second and higher order terms of $1 / N$, we get

$N_{\mathrm{e}}=\frac{9(4-3 \beta) N}{4\left(4-4 \beta+2 \sigma^{2}+4 \sigma_{\mathrm{f}}^{2}-\beta \sigma_{\mathrm{m}}^{2}-2 \beta \sigma_{\mathrm{f}}^{2}\right)}$.

For random mating, eqn 16 reduces to

$N_{\mathrm{e}}=\frac{9 N}{4+2 \sigma^{2}+4 \sigma_{\mathrm{f}}^{2}}$

approximately, which was also obtained by Pollak (1990) following a variance of drift derivation and by Caballero (1995) and Nagylaki (1995) following different inbreeding approaches. Eqn 17 is different from an expression of Moran \& Watterson (1959), Ethier \& Nagylaki (1980) and Nagylaki (1981), and the reason for the difference is discussed by Caballero (1995) and Nagylaki (1995).

For an infinite population with partial full-sib mating, the inbreeding coefficient at equilibrium or the deviation from Hardy-Weinberg proportions is $F_{\text {IS }}=\alpha=\beta /(4-3 \beta)$. Substituting this relation into eqn 16 , we obtain a more general expression

$N_{\mathrm{e}}=\frac{9 N}{4(1-\alpha)+2(1+3 \alpha) \sigma^{2}+4(1+\alpha) \sigma_{\mathrm{f}}^{2}-4 \alpha \sigma_{\mathrm{m}}^{2}}$.

For a finite population, even if mating is at random, the value of $\alpha$ is not exactly zero because of the difference in gene frequencies of the two independent gamete samples, one from male parents and the other from female parents. The expression for $\alpha$ in random mating populations of equal numbers $(N / 2)$ of males and females is
$\alpha_{\mathrm{R}}=-1 /(N-1)$ approximately. Thus for a small population with partial full-sib mating, $\alpha=\beta /(4-3 \beta)-1 /(N-1)$ should be substituted into eqn 18 to get a more satisfactory prediction of $N_{\mathrm{e}}$.

Although eqn 18 is derived for a partial full-sib mating population, it applies to other nonrandom mating populations as well. The inbreeding coefficient at equilibrium for an X-linked locus in populations under some partial inbreeding systems has been given earlier in this paper.

\section{General equations for effective size}

In this section we remove the restriction of equal numbers of males and females. We assume that the population consists of $N_{\mathrm{m}}$ males and $N_{\mathrm{f}}$ females in each generation $\left(N_{\mathrm{m}}<N_{\mathrm{f}}\right)$, and each male mates with an equal number of females $\left(R=N_{\mathrm{f}} / N_{\mathrm{m}}\right)$. In such a case, it is not feasible to derive complete recurrence equations for the inbreeding coefficient for nonrandom mating populations. However, following the variance of change in gene frequency approach similar to that of Latter (1959), Hill (1979), Pollak (1990) and Caballero \& Hill (1992), we can derive general equations for effective size.

Males heterogametic First we consider species where males are the heterogametic sex. Letting $x_{\mathrm{m} i}$ (=0 or 1$)$ and $x_{\mathrm{fij}}(=0,1 / 2$ or 1$)$ be the gene frequencies of male $i$ and its mate $j(j=1$ to $R)$, the gene frequency in generation $t-1$ is

$q=\frac{1}{3 N_{\mathrm{m}}} \sum_{i=1}^{N_{\mathrm{m}}} x_{\mathrm{m} i}+\frac{2}{3 N_{\mathrm{f}}} \sum_{i=1}^{N_{\mathrm{m}}} \sum_{j=1}^{R} x_{\mathrm{fij} j}$.

If the numbers of male and female offspring contributed by the $j$ th female parent mated with the $i$ th male parent are $n_{\mathrm{fmij}}$ and $n_{\mathrm{ffi} j}$, respectively, then the gene frequency in generation $t$ is

$$
\begin{aligned}
& Q=\frac{1}{3 N_{\mathrm{m}}} \sum_{i=1}^{N_{\mathrm{m}}} \sum_{j=1}^{R}\left[n_{\mathrm{fmij}} x_{\mathrm{f} i j}+\sum_{l=1}^{n_{\mathrm{fmij}}} \delta_{\mathrm{f} i j l}\right] \\
& +\frac{2}{3 N_{\mathrm{f}}} \sum_{i=1}^{N_{\mathrm{m}}} \sum_{j=1}^{R}\left[n_{\mathrm{ffij}}\left(\frac{x_{\mathrm{m} i}+x_{\mathrm{fij}}}{2}\right)+\frac{1}{2} \sum_{l=1}^{n_{\mathrm{f} i j}} \delta_{\mathrm{fijl}}\right] \\
& =\frac{1}{3 N_{\mathrm{m}}} \sum_{i=1}^{N_{\mathrm{m}}} \sum_{j=1}^{R}\left[n_{\mathrm{fmij}} x_{\mathrm{fij}}+\sum_{l=1}^{n_{\mathrm{fm} i j}} \delta_{\mathrm{fij} l}\right] \\
& \frac{1}{3 N_{\mathrm{f}}} \sum_{i=1}^{N_{\mathrm{m}}}\left[n_{\mathrm{m} f i} x_{\mathrm{m} i}+\sum_{j=1}^{R} n_{\mathrm{ffij}} x_{\mathrm{fij}}+\sum_{j=1}^{R} \sum_{l=1}^{n_{\mathrm{ffij}}} \delta_{\mathrm{fijl}}\right] \text {, }
\end{aligned}
$$


where

$n_{\mathrm{m} f i}=\sum_{j=1}^{R} n_{\mathrm{ffij}}$

is the number of female offspring contributed by male parent $i, \delta_{\mathrm{fijl}}$ is the difference in gene frequency between the $l$ th sampled gene coming from the $j$ th female parent mated with male $i$ and its frequency $x_{\mathrm{f} i j}$ in that individual. Clearly, the value of $\delta_{\mathrm{f} j \mathrm{j} l}$ is 0 if the parent is a homozygote or $\pm 1 / 2$ with equal probabilities if the parent is a heterozygote.

The genetic drift in gene frequency is $\Delta q=q-Q$. Inasmuch as the gene frequency of an individual and the number of offspring of the same individual or a different individual are independently distributed, as are $\delta_{\text {fikl }}$ and other terms, we obtain the variance in gene frequency drift, $\sigma_{\Delta q}^{2}$, from eqns $19 \mathrm{a}$ and $19 \mathrm{~b}$ as

$$
\begin{aligned}
\sigma_{\Delta q}^{2}= & V(q-Q) \\
= & \frac{1}{9 N_{\mathrm{m}}^{2}}\left[N_{\mathrm{f}} \sigma_{\mathrm{fm}}^{2} V\left(x_{\mathrm{fij}}\right)+N_{\mathrm{m}} V\left(\delta_{\mathrm{f} i j l}\right)\right] \\
& +\frac{1}{9 N_{\mathrm{f}}^{2}}\left[N_{\mathrm{m}} \sigma_{\mathrm{mf}}^{2} V\left(x_{\mathrm{m} i}\right)+2 N_{\mathrm{f}} \operatorname{Cov}(m f, f f)\right. \\
& \left.\times \operatorname{Cov}\left(x_{\mathrm{m} i}, x_{\mathrm{fij}}\right)+N_{\mathrm{f}} \sigma_{\mathrm{ff}}^{2} V\left(x_{\mathrm{fij}}\right)+N_{\mathrm{f}} V\left(\delta_{\mathrm{fij} i}\right)\right] \\
& +\frac{2}{9 N_{\mathrm{m}} N_{\mathrm{f}}}\left[N_{\mathrm{f}} \operatorname{Cov}(m f, f m) \operatorname{Cov}\left(x_{\mathrm{m} i}, x_{\mathrm{fij}}\right)\right. \\
& \left.+N_{\mathrm{f}} \operatorname{Cov}(f m, f f) V\left(x_{\mathrm{fij}}\right)\right],
\end{aligned}
$$

where $\operatorname{Cov}(p q, r s)$ is the covariance between the numbers of offspring of sexes $q$ and $s$ from parents of sexes $p$ and $r$ respectively ( $p, q, r, s=m$ or $f$ ). If $p=r$, they represent the same parent, otherwise they are mated parents.

It can be shown that the variances in gene frequency of males and females are $V\left(x_{\mathrm{m} i}\right)=q(1-q)$ and $V\left(x_{\mathrm{fij}}\right)=q(1-q)\left(1+\alpha_{I}\right) / 2$, respectively, where $\alpha_{I}$ is the departure from Hardy-Weinberg proportions in female individuals. The covariance between gene frequencies of the parents is $\operatorname{Cov}\left(x_{\mathrm{m} i}, x_{\mathrm{fij}}\right)$ $=q(1-q) \alpha_{o}$, where $\alpha_{o}$ is the correlation between genes in mated pairs of parents. The variance attributable to Mendelian segregation, $V\left(\delta_{\mathrm{ij} j}\right)$, equals the product of the frequency of heterozygotes, $2 q(1-q)\left(1-\alpha_{l}\right)$, and the variance generated from them, $1 / 4$; thus $V\left(\delta_{\mathrm{fijl}}\right)=\frac{1}{2} q(1-q)\left(1-\alpha_{l}\right)$. Substituting these relations into eqn $19 \mathrm{c}$ and equating $19 \mathrm{c}$ to the drift variance per generation in the ideal population, $q(1-q) /\left(2 N_{\mathrm{e}}\right)$, we therefore obtain

$$
\begin{aligned}
\frac{1}{N_{\mathrm{e}}}=\frac{1}{9 N_{\mathrm{m}}} & {\left[1-\alpha_{I}+4 \alpha_{o} \operatorname{Cov}(m f, f m)\right.} \\
+ & \left.2\left(1+\alpha_{I}\right) \operatorname{Cov}(f m, f f)+2\left(\frac{N_{\mathrm{m}}}{N_{\mathrm{f}}}\right)^{2} \sigma_{\mathrm{mf}}^{2}\right] \\
& +\frac{1}{9 N_{\mathrm{f}}}\left[1-\alpha_{I}+4 \alpha_{o} \operatorname{Cov}(m f, f f)+\left(1+\alpha_{I}\right) \sigma_{\mathrm{ff}}^{2}\right. \\
& \left.+\left(1+\alpha_{l}\right)\left(\frac{N_{\mathrm{f}}}{N_{\mathrm{m}}}\right)^{2} \sigma_{\mathrm{fm}}^{2}\right] .
\end{aligned}
$$

Because the difference between $\alpha_{I}$ and $\alpha_{o}$ is rather small, we take $\alpha_{I} \approx \alpha_{o} \approx \alpha$ for simplicity in the following discussion. For a large population with random mating, $\alpha=0$, and eqn 20 reduces to

$$
\begin{aligned}
\frac{1}{N_{\mathrm{e}}}= & \frac{1}{9 N_{\mathrm{m}}}\left[1+2\left(\frac{N_{\mathrm{m}}}{N_{\mathrm{f}}}\right)^{2} \sigma_{\mathrm{mf}}^{2}\right]+\frac{1}{9 N_{\mathrm{f}}} \\
& \times\left[1+\sigma_{\mathrm{ff}}^{2}+2\left(\frac{N_{\mathrm{f}}}{N_{\mathrm{m}}}\right) \operatorname{Cov}(f m, f f)+\left(\frac{N_{\mathrm{f}}}{N_{\mathrm{m}}}\right)^{2} \sigma_{\mathrm{fm}}^{2}\right],
\end{aligned}
$$

which was derived by Pollak $(1980,1990)$ and Caballero (1995) by different methods. For the special case of a Poisson distribution of family size, from eqn 21 we get the classical result (Wright, 1933; Malécot, 1951; Kimura, 1963)

$N_{\mathrm{e}}=\frac{9 N_{\mathrm{m}} N_{\mathrm{f}}}{4 N_{\mathrm{m}}+2 N_{\mathrm{f}}}$.

For a population of equal numbers of males and females, we have $N_{\mathrm{f}}=N_{\mathrm{m}}=N / 2, \sigma_{\mathrm{mf}}^{2}=\sigma_{\mathrm{ff}}^{2}=\operatorname{Cov}(m f$, $f f)=\sigma_{\mathrm{f}}^{2}, \sigma_{\mathrm{fm}}^{2}=\sigma_{\mathrm{m}}^{2}, \operatorname{Cov}(m f, f m)=\operatorname{Cov}(f m, f f)=0$, and eqn 20 is simplified to eqn 18 as expected.

If the numbers of male and female offspring from each female parent are of independent Poisson distributions, then we have $\operatorname{Cov}(m f, f f)=\sigma_{\mathrm{ff}}^{2}=1$, $\operatorname{Cov}(m f, f m)=\operatorname{Cov}(f m, f f)=0$ and $\sigma_{\mathrm{mf}}^{2}=1 / \sigma_{\mathrm{fm}}^{2}=N_{\mathrm{f}} /$ $N_{\text {m }}$, and eqn 20 reduces to

$N_{\mathrm{e}}=\frac{9 N_{\mathrm{m}} N_{\mathrm{f}}}{4(1+\alpha) N_{\mathrm{m}}+2 N_{\mathrm{f}}}$.

In a population of different numbers of males and females, minimal inbreeding can be achieved by choosing as parents one female and a probability of $N_{\mathrm{m}} / N_{\mathrm{f}}$ of having one male from each female's progeny, with the restriction that each male contri- 
butes one son to the next generation (Gowe et al., 1959). In this situation, the numbers of male offspring from different female parents are no longer of the same and independent distributions, $\sigma_{\mathrm{fm}}^{2}=\left(N_{\mathrm{m}} / N_{\mathrm{f}}\right)\left(1-N_{\mathrm{m}} / N_{\mathrm{f}}\right), \operatorname{Cov}(m f, f m)=\operatorname{Cov}(f m, f f)$ $=\operatorname{Cov}(m f, f f)=\sigma_{\mathrm{mf}}^{2}=\sigma_{\mathrm{ff}}^{2}=0$, and eqn 20 reduces to

$N_{\mathrm{e}}=\frac{9 N_{\mathrm{m}} N_{\mathrm{f}}}{2\left(N_{\mathrm{f}}-\alpha N_{\mathrm{m}}\right)}$.

Females heterogametic For species where females are the heterogametic sex, as in poultry, the same population structure and mating system gives (using the same procedure shown above)

$$
\begin{aligned}
\frac{1}{N_{\mathrm{e}}}=\frac{1}{9 N_{\mathrm{m}}} & {\left[1-\alpha_{I}+\left(1+\alpha_{I}\right) \sigma_{\mathrm{mm}}^{2}+2\left(1+\alpha_{I}\right)\left(\frac{N_{\mathrm{m}}}{N_{\mathrm{f}}}\right)\right.} \\
& \left.\times \operatorname{Cov}(m m, m f)+\left(1+\alpha_{I}\right)\left(\frac{N_{\mathrm{m}}}{N_{\mathrm{f}}}\right)^{2} \sigma_{\mathrm{mf}}^{2}\right] \\
& +\frac{1}{9 N_{\mathrm{f}}}\left[1-\alpha_{I}+4 \alpha_{o}\left(\frac{N_{\mathrm{f}}}{N_{\mathrm{m}}}\right)^{2} \operatorname{Cov}(m m, f m)\right. \\
& \left.+2\left(\frac{N_{\mathrm{f}}}{N_{\mathrm{m}}}\right)^{2} \sigma_{\mathrm{fm}}^{2}+4 \alpha_{o}\left(\frac{N_{\mathrm{f}}}{N_{\mathrm{m}}}\right) \operatorname{Cov}(m f, f m)\right],
\end{aligned}
$$

where $\alpha_{I}$ is the departure from Hardy-Weinberg proportions in male individuals. When $N_{\mathrm{f}}=N_{\mathrm{m}}=N /$ 2 , eqn 25 also reduces to eqn 18 as expected. For a large population with random mating, $\alpha_{I}=\alpha_{o}=0$, eqn 25 reduces to eqn 21 replacing $m$ and $f$ for $f$ and $m$, respectively. For cases of Poisson distribution of family size and minimal inbreeding in a large population with random mating, eqn 25 reduces to

$N_{\mathrm{e}}=\frac{9 N_{\mathrm{m}} N_{\mathrm{f}}}{4 N_{\mathrm{f}}+2 N_{\mathrm{m}}}$

and

$N_{\mathrm{e}}=\frac{9 N_{\mathrm{m}} N_{\mathrm{f}}}{3 N_{\mathrm{f}}-N_{\mathrm{m}}}$,

respectively. Eqn 27 was also derived by Caballero (1995).

For independent Poisson distribution of family size and minimal inbreeding, eqn 25 reduces to
$N_{\mathrm{e}}=\frac{9 N_{\mathrm{m}} N_{\mathrm{f}}}{4(1+\alpha) N_{\mathrm{f}}+2 N_{\mathrm{m}}}$

and

$N_{\mathrm{e}}=\frac{9 N_{\mathrm{m}} N_{\mathrm{f}}}{3(1+\alpha) N_{\mathrm{f}}-(1+5 \alpha) N_{\mathrm{m}}}$,

respectively.

\section{Discussion}

In nonrandom mating populations, effective size is defined as the asymptotic value (over time) of the rate of increase in inbreeding. The larger the departure from random mating, the more generations are required before the asymptotic rate of inbreeding is attained. In practice, most populations do not maintain the same characteristics for such a long time, and in breeding programmes interest is more likely to be concentrated on early generations. Thus, the recurrence equations for the inbreeding coefficient derived in this paper are useful for an exact prediction of inbreeding.

To consider the effect of the exclusion of full-sib mating on effective size, terms involving $1 / N^{2}$ should not be omitted in deriving equations for $N_{\mathrm{e}}$. A general expression for $N_{\mathrm{e}}$ derived from eqn 9, taking $1 / N^{2}$ into consideration, is too complicated and not given here. I only consider the four special cases given in eqns $11,12,14$ and 15 . The effective sizes for random mating and nonsib mating are $N_{\mathrm{e}}=\frac{3}{4} N+\frac{2}{3}$ and $N_{\mathrm{e}}=\frac{3}{4} N+\frac{11}{12}$, respectively, under random selection, $N_{\mathrm{e}}=\frac{9}{4} N-\frac{5}{4}$ and $N_{\mathrm{e}}=\frac{9}{4} N-\frac{19}{8}$, respectively, under equal family selection. Excluding sib matings increases $N_{\mathrm{e}}$ by a value of $1 / 4$ under random selection and decreases $N_{\mathrm{e}}$ by $9 / 8$ under equal family selection compared with random mating, irrespective of the census size. These results are slightly different from those found for autosomal genes. For the case of random selection and full-sib mating exclusion, the equation derived by Wright (1951), Robinson \& Bray (1965) and Jacquard (1971) is $N_{\mathrm{e}}=N+2$, which has been shown to be incorrect and the corresponding equation should be $N_{\mathrm{e}}=N+1$ (Wang, 1995). Thus excluding full-sib mating increases $N_{\mathrm{e}}$ by a value of $1 / 2\left(N_{\mathrm{e}}=N+1 / 2\right.$ for random mating) under random selection, whereas it decreases $N_{\mathrm{e}}$ by a value of one under equal family size selection for autosomal genes (Robinson \& Bray, 1965; Wang, 1995). The effect of sib mating exclusion on $N_{\mathrm{e}}$ (compared with random mating) is slightly smaller under random selection 
and slightly larger under equal family size selection for X-linked genes than for autosomal genes.

For X-linked loci, males and females are of different importance in determining the inbreeding and effective size. This can be clearly seen from eqn 17. For example, if the number of male progeny from each family is Poisson distributed and that of female progeny is equal for each family, then $\sigma_{\mathrm{m}}^{2}=\sigma^{2}=1, \quad \theta=\sigma_{\mathrm{f}}^{2}=0$ and $N_{\mathrm{e}}=\frac{3}{2} N$, whereas the opposite stipulation leads to $N_{\mathrm{e}}=\frac{9}{10} N$. Thus to keep minimal inbreeding in control populations, special stress should be laid on minimizing the variation in the number of female progeny per family.

It is interesting to compare eqns 23 and 24 with the corresponding expressions for autosomal genes, which are

$N_{\mathrm{e}}=\frac{4 N_{\mathrm{m}} N_{\mathrm{f}}}{(1+\alpha)\left(N_{\mathrm{f}}+N_{\mathrm{m}}\right)}$

(Caballero \& Hill, 1992; Wang, 1996) and

$N_{\mathrm{e}}=\frac{16 N_{\mathrm{m}} N_{\mathrm{f}}}{(3-\alpha) N_{\mathrm{f}}+(1-3 \alpha) N_{\mathrm{m}}}$

(Wang, 1996), respectively. For a Poisson distribution of family size, it can be found that effective size for X-linked genes is smaller than that for autosomal genes unless

$N_{\mathrm{f}}>\frac{7(1+\alpha) N_{\mathrm{m}}}{1+9 \alpha}$ when $\alpha>-\frac{1}{9}$.

The smaller the value of $\alpha$, the larger is the sex ratio required for the effective size for an X-linked locus to be greater than that for an autosomal locus. When $\alpha \leq-\frac{1}{9}$, which may occur in a small population with avoidance of close inbreeding, effective size for an $\mathrm{X}$-linked locus is always smaller than that for an autosomal locus. For minimal inbreeding, the situation is reversed. The effective size for X-linked genes is larger than that for autosomal genes unless

$N_{\mathrm{f}}>\frac{(9+5 \alpha) N_{\mathrm{m}}}{5+9 \alpha}$.

From eqn 24 it can be seen that the smaller the number of females $\left(N_{\mathrm{f}}>N_{\mathrm{m}}\right)$, the larger the effective size when $\alpha>0$. When $\alpha=0, N_{\mathrm{e}}=9 N_{\mathrm{m}} / 2$, independently of the number of females (Caballero, 1995). When $\alpha<0$, the value of $N_{\mathrm{e}}$ increases with $N_{\mathrm{f}}$. Thus for minimal inbreeding to be attained for an $\mathrm{X}$-linked locus, as few females as possible should be used if close inbreeding, which results in a positive value of $\alpha$, is carried out.
A comparison between eqns 28 and 29 with eqns 23 and 24 indicates that the effective size for populations with heterogametic males is always larger than that with heterogametic females when $N_{\mathrm{f}}>N_{\mathrm{m}}$. This is because there are more $\mathrm{X}$-linked genes $\left(2 N_{\mathrm{f}}+N_{\mathrm{m}}\right)$ for the first case than that for the second $\left(N_{\mathrm{f}}+2 N_{\mathrm{m}}\right)$. Comparing eqns 28 and 29 with the corresponding expressions for autosomal genes, we find that effective size for an X-linked locus is always smaller than that for an autosomal locus for a Poisson distribution of family size, and when $N_{\mathrm{f}}>N_{\mathrm{m}}$ for minimal inbreeding. From eqn 29 it can be seen that $N_{\mathrm{e}}$ is a monoincreasing and monodecreasing function of $N_{\mathrm{f}}$ when $\alpha<-1 / 5$ and $\alpha>-1 / 5$, respectively. When $\alpha=-1 / 5, N_{\mathrm{e}}$ is independent of the value of $N_{\mathrm{f}}$.

The results for effective size for an X-linked locus in a population with minimal inbreeding are interesting and perhaps important from a practical point of view. For large random mating populations $(\alpha=0)$, Caballero (1995) concluded that effective size is independent of the number of females for populations where males are the heterogametic sex and that increasing the number of females may actually decrease effective size for populations where the heterogametic sex is female. The results in this paper indicate that, in nonrandom mating populations, as few females as possible for each male should be used for maximum effective size when $\alpha>0$ and $\alpha>-1 / 5$ where the heterogametic sex is male and female, respectively.

\section{Acknowledgements}

I am grateful to two anonymous referees for useful comments on the manuscript.

\section{References}

CABAllero, A. 1994. Developments in the prediction of effective population size. Heredity, 73, 657-679.

CABAllero, A. 1995. On the effective size of populations with separate sexes, with particular reference to sex-linked genes. Genetics, 139, 1007-1011.

CABAllero, A. AND HILl, w. G. 1992. Effective size of nonrandom mating populations. Genetics, 130, 909-916.

ETHIER, S. N. AND NAGYLAK1, T. 1980. Diffusion approximations of Markov chains with two time scales and applications to population genetics. Adv. Appl. Prob., 12, 14-49.

FALCONER, D. S. 1989. Introduction to Quantitative Genetics, 3rd edn. Longman, Harlow, Essex.

GHAl, G. L. 1969. Structure of populations under mixed random and sib mating. Theor. Appl. Genet., 39, 179-182.

(c) The Genetical Society of Great Britain, Heredity, 76, 569-577. 
GOWE, R. S., ROBERTSON, A. AND LATTER, B. D. H. 1959. Environment and poultry breeding problems. 5. The design of poultry control strains. Poultry Sci., 38, $462-471$.

HeDrick, P. W. AND COCKerham, c. c. 1986. Partial inbreeding: equilibrium heterozygosity and the heterozygosity paradox. Evolution, 40, 856-861.

HILL, w. G. 1979. A note on effective population size with overlapping generations. Genetics, 92, 317-322.

JACQUARD, A. 1971. Effect of exclusion of sib-mating on genetic drift. Theor. Pop. Biol., 2, 91-99.

KEMPTHORne, O. 1957. An Introduction to Genetic Statistics. John Wiley and Sons, New York.

KIMURA, M. 1963. A probability method for treating inbreeding systems, especially with linked genes. Biometrics, 19, 1-17.

LATTER, B. D. H. 1959. Genetic sampling in a random mating population of constant size and sex ratio. Aust. J. Biol. Sci., 12, 500-505.

LI, c. c. 1976. First Course in Population Genetics. Boxwood, Pacific Grove, CA.

MALÉCOT, G. 1948. Les Mathématiques de l'hérédité. Masson et Cie, Paris.

MALÉCOT, G. 1951. Un traitment stochastique des problèmes linéaires (mutation, linkage, migration) en génétique de population. Ann. Univ. Lyon, Sci., Sec. A, 14, 79-117.

MORAN, P. A. P. AND WATTERSON, G. A. 1959. The genetic effects of family structure in natural populations. Aust. J. Biol. Sci., 12, 1-5.
NAGYLAKI, T. 1981. The inbreeding effective population number and the expected homozygosity for an X-linked locus. Genetics, 97, 731-737.

NAGYLAKI, T. 1995. The inbreeding effective population number in dioecious populations. Genetics, 139, $473-485$

PLUM, M. 1954. Computation of inbreeding and relationship coefficients. J. Hered., 45, 92-94.

POLLAK, E. 1980. Effective population numbers and mean times to extinction in dioecious populations with overlapping generations. Math. Biosci., 52, 1-25.

POLLAK, E. 1990. The effective population size of an agestructured population with a sex-linked locus. Math. Biosci., 101, 121-130.

ROBINSON, P. AND BRAY, D. F. 1965. Expected effects on the inbreeding and rate of gene loss of four methods of reproducing finite diploid populations. Biometrics, $\mathbf{2 1}$, 447-458.

WANG, J. 1995. Exact inbreeding coefficient and effective size in finite populations under partial sib mating. Genetics, 140, 357-363.

WANG, J. 1996. Inbreeding and variance effective sizes for nonrandom mating populations. Evolution (in press).

wright, s. 1933. Inbreeding and homozygosis. Proc. Natl. Acad. Sci., U.S.A., 19, 411-420.

WRIGHT, s. 1951. The genetical structure of populations. Ann. Eugen., 15, 323-354.

Wright, s. 1969. Evolution and the Genetics of Populations, vol. 2, The Theory of Gene Frequencies. The University of Chicago Press, Chicago, IL. 